Mecánica Computacional Vol. XXI, pp. 2776-2790

S. R. Idelsohn, V. E. Sonzogni and A. Cardona (Eds.)

Santa Fe-Paraná, Argentina, October 2002

\title{
STRUCTURAL SHAPE OPTIMISATION USING BOUNDARY ELEMENTS AND THE BIOLOGICAL GROWTH METHOD
}

\author{
Carolina Wessel $^{1}$, Adrián P. Cisilino ${ }^{2}$ and Berardi Sensale ${ }^{3}$ \\ ${ }^{1}$ Facultad de Ingeniería, Universidad Austral \\ Garay 125, Ciudad Autónoma de Buenos Aires, Argentina. \\ e-mail: cwessel@fi.mdp.edu.ar \\ ${ }^{2}$ Facultad de Ingeniería, Universidad Nacional de Mar del Plata - CONICET \\ Av. Juan B. Justo 4302 (7600) Mar del Plata, Argentina. \\ ${ }^{3}$ Instituto de Estructuras y Transporte, Facultad de Ingeniería, Universidad de la República \\ J. Herrera y Reissig $665-11300$ Montevideo, Uruguay
}

Key words: Shape Optimization, Biological Growth Method, Boundary Elements Method, Dual Reciprocity Method.

\begin{abstract}
A numerical evolutionary procedure for the structural optimisation for stress reduction of two-dimensional structures is presented in this paper. The proposed procedure couples the Biological Growth Method (BGM) with the Boundary Element Method (BEM). The boundary-only intrinsic characteristic of BEM together with its accuracy in the boundary displacement and stress solutions, make boundary elements especially attractive for solving shape optimisation problems. Two formulations of BEM are used in this work: the standard for two-dimensional elastostatics for the stress analysis, and the Dual Reciprocity Method (DRM) which is used to model the swelling or shrinking of the material. Two examples are analysed to illustrate the proposed methodology and to demonstrate its versatility and robustness.
\end{abstract}




\section{INTRODUCTION}

Locally high stresses frequently give place to the failure of structures under service conditions, thus reducing service life. Consequently structural shape optimisation for stress reduction has been so far a major issue, examined both with analytical and numerical tools, as reviewed by Belegundu and Chandrupatla ${ }^{\mathrm{i}}$.

Biological structures give excellent examples for this purpose, as they in fact optimise their shape changing their contour to adapt to external loads while reducing stress peaks. Mattheck ${ }^{\mathrm{ii}}$ observed this behaviour and consequently introduced the Biological Growth Method (BGM). He defines optimum shape as the one that shows a state of constant stress at part of or the whole of the surface of the structure. The process of self-optimisation (in tree butts, branch joints, deer antlers) is carried out through the swelling or shrinking of the soft outermost layer of material, process that reduces local stress levels.

Since the pioneer work by Mattheck and Burkhardt ${ }^{\mathrm{iii}}$ was published interest has been shown in BGM coupled with the Finite Element Method (FEM) (e. g. Chen and Tsaiv, Tekkaya and Güneriv ${ }^{\mathrm{v}}$, Li et $a l^{\mathrm{vi}}$. and Chaperon ${ }^{\mathrm{vii}}$ ). Meanwhile the Boundary Element Method (BEM) has become a popular alternative in structural shape optimisation (e. g. Baron and Yang $^{\text {viii }}$, Kane and Saigal ${ }^{\mathrm{x}}$ and Mellings and Aliabadi ${ }^{\mathrm{x}}$ ) due to its accuracy in the boundary displacement and stress solutions as well as the fact that remeshing is easier for BEM than for FEM. Since BGM considers that the swelling or shrinking of only the soft outermost layer of material governs the optimisation process BEM becomes especially attractive for solving shape optimisation problems using Mattheck's approach. As far as the authors know there is only one published paper dedicated to coupling BGM and BEM, Cai et al $l^{\mathrm{xi}}$. However, in this work the swelling or shrinking of the material is extended to the complete geometry and not only to the boundary layer as originally proposed by Mattheck.

The swelling or shrinking of the soft thin outermost layer can be described by means of a thermoelastic equation, mainly replacing temperature fields by stress distributions as already suggested by Mattheck and Moldenhauer ${ }^{x i i}$. Thermal effects (as much as body forces) were an initial restriction in the use of BEM as they must be included in the formulation by means of a domain integral, thus loosing the method its original "boundary-only" character. Many different approaches have been developed to overcome this drawback, among which the Dual Reciprocity Method (DRM) has become widely used. The implementation proposed in this work, then, makes use of two BEM formulations: the standard for two-dimensional elastostatics, which is used for the stress analysis of the problem; and the Dual Reciprocity Method (DRM) to model swelling.

The authors have accomplished an evolutionary numerical algorithm that couples BGM and BEM (both standard and DRM formulations), by means of exponential splines, which provide easy remeshing. This algorithm is presented in this paper together with two examples that confirm the robustness of the combination. 


\section{THE BIOLOGICAL GROWTH METHOD}

The Biological Growth Method (BGM) was first introduced by Mattheck ${ }^{\text {ii }}$. Based on his observations in Nature (tree butts, branch joints, deer antlers, etc.) he posits that biological structures always self-optimise their shapes according to natural external loads. He defines optimum shape as the one that shows a state of constant stress at part of or the whole of the surface of the component. The process of self-optimisation consists of the swelling or the shrinking of the soft outermost layer of material, following the law

$$
\varepsilon_{v}=k\left(\sigma_{v m}-\sigma_{r e f}\right)
$$

where $\varepsilon_{v}$ is the volumetric swelling strain rate, which is stated to be proportional to a driving function given by the difference from von Mises stress $\left(\sigma_{v m}\right)$ and a reference stress $\left(\sigma_{r e f}\right)$, an expected value. This equation holds for each point in the optimisation domain. According to (1), if $\sigma_{v m}-\sigma_{r e f}>0$ the thin layer swells, while, if $\sigma_{v m}-\sigma_{r e f}<0$ the thin layer shrinks, in complete agreement with Mattheck's observations in Nature. An elegant method to implement (1) is by means of a thermal expansion analogy based on the generalized Hooke's law, where $\left(\sigma_{v m}-\right.$ $\left.\sigma_{r e f}\right)$ is replaced by the change in temperature $\theta$, according to the following equation

$$
\alpha \theta=\gamma k\left(\sigma_{v m}-\sigma_{r e f}\right)
$$

where $\alpha$ is the thermal expansion coefficient, and $\gamma$ is a units conversion factor. Although this is not the only possible approach, it was the strategy followed in this work.

The method can be summarised as follows:

1. Generate a reasonable design, including the thin layer having a small Young modulus in the optimisation domain;

2. Choose an appropriate stress reference value $\left(\sigma_{r e f}\right)$;

3. Perform an elastic static load case to get the von Mises stress distribution $\left(\sigma_{v m}\right)$ over the whole structure;

4. Perform a thermal expansion analysis with a temperature field $\theta$ given by (2). This computation supplies the displacements $u(x, y)$ and $v(x, y)$ along the optimisation boundary.

5. Update the optimisation boundary by

$$
\begin{aligned}
& x^{\prime}=x+C \cdot u(x, y) \\
& y^{\prime}=y+C \cdot v(x, y)
\end{aligned}
$$

where the $C$ is a magnification factor put to use to accelerate convergence.

Steps 3 to 5 are repeated until von Mises stresses are reduced to the reference value or design limitations restrain further changes in the geometry.

Notice that $\gamma, \alpha, k$ and $C$ have similar effects on the results: they all act as magnification factors. Therefore only one of them is actually needed. In this work only $C$ is considered, 
whereas $\gamma$ and $k$ are taken as unity and $\alpha$ as the actual thermal expansion coefficient of the material. Special attention should be taken to choose suitable values of $\sigma_{r e f}$ and $C$. Otherwise the whole process of optimisation may not converge ${ }^{\text {iii }}$.

\section{THE BOUNDARY ELEMENT METHOD}

A brief description of the Boundary Element Method (BEM) is given here in order to point out the different formulations used in this work. For further details on BEM the reader should refer to the books by Brebbia and Dominguez ${ }^{\text {xiii }}$ and Partridge $e t a l^{x i v}$.

\subsection{BEM for two-dimensional elasticity}

The formulation of BEM for two-dimensional elasticity problems starts with the Navier equation

$$
G u_{j, k k}+\frac{G}{1-2 v} u_{k, k j}+b_{j}=0
$$

where $j, k$ denote Cartesian components, $G$ is the shear modulus, $v$ is the Poisson ratio, $b_{j}$ are the components of body forces and $u_{k}$ are the displacements.

After including boundary conditions on traction and displacement fields where they correspond and using mathematical standard procedures (Aliabadi and Rooke $\mathrm{xv}^{\mathrm{xv}}$ ), a boundary integral and domain integral equation is obtained:

$$
c_{l k}^{i}\left(x^{\prime}\right) u_{k}^{i}\left(x^{\prime}\right)+\int_{\Gamma} p_{l k}^{*}\left(x^{\prime}, x\right) u_{k}(x) d \Gamma(x)=\int_{\Gamma} u_{l k}^{*}\left(x^{\prime}, x\right) p_{k}(x) d \Gamma(x)+\int_{\Omega} u_{l k}^{*}\left(x^{\prime}, x\right) b_{k}(x) d \Omega
$$

which relates displacements $u_{i}$ at the collocation point $x^{\prime}$ with the displacements $u_{k}$ and tractions $p_{k}$ in the boundary $\Gamma$ and the body forces $b_{k}$; and $p_{l k}^{*}\left(x^{\prime}, x\right)$ and $u_{l k}^{*}\left(x^{\prime}, x\right)$ represent the traction and displacement fundamental solutions at a boundary point $x$ due to a unit load placed at location $x^{\prime}$. The term $c_{l k}\left(x^{\prime}\right)$ is generally a function of the geometry variation at the boundary point $x^{\prime}$. Providing that $x^{\prime}$ is a smooth boundary point, that is, the outward normal vector to the boundary is continuous at $x^{\prime}$, then it can be shown that $c_{l k}\left(x^{\prime}\right)=1 / 2 \delta_{l k}$ (Aliabadi and Rooke $^{\mathrm{xv}}$ ).

To solve this equation numerically, the model contour is discretized into $N$ elements, where displacements $u_{k}(x)$ and tractions $p_{k}(x)$ are expressed in terms of the nodal values $u_{k}^{i}$ and $p_{k}^{i}$ by means of isoparametric interpolation functions $\phi^{i}$.

Once this discretized boundary integral equation is applied to all collocation points and in absence of external loads, a set of equations results which can be expressed in matrix form as

$$
\mathbf{H u}=\mathbf{G p}
$$


where $\mathbf{H}$ and $\mathbf{G}$ are $N \times N$ matrices and $\mathbf{u}$ and $\mathbf{p}$ are vectors of length $N$ containing the nodal values $u_{k}^{i}$ and $p_{k}^{i}$ respectively. The unknown nodal values of $u_{k}^{i}$ and $p_{k}^{i}$ can be recovered solving the system (6) after replacing the boundary conditions.

\subsection{The Dual Reciprocity BEM (DRM) for two-dimensional thermoelasticity.}

Thermal effects (as much as body forces) were an initial restriction in the use of BEM as they must be included in the formulation by means of a domain integral (see (5)), thus loosing the method its original "boundary-only" character. Many different approaches have been developed to overcome this problem, among which the Dual Reciprocity Method (DRM) has become widely used. The basic idea behind this approach is to adopt particular solutions and global approximation functions.

Following Partridge and Sensale ${ }^{\mathrm{xvi}}$ the effects produced by changes in temperature $\theta$ in elastic bodies can be represented by initial stresses $\sigma^{0}$, such that:

$$
\sigma_{j k}^{0}=\chi \theta \delta_{j k}
$$

where $\chi=-2 G \frac{1+v}{1-2 v} \alpha$, which transforms (5) into

$$
c_{l k}^{i}\left(x^{\prime}\right) u_{k}^{i}\left(x^{\prime}\right)+\int_{\Gamma} p_{l k}^{*}\left(x^{\prime}, x\right) u_{k}(x) d \Gamma(x)=\int_{\Gamma} u_{l k}^{*}\left(x^{\prime}, x\right) p_{k}(x) d \Gamma(x)-\int_{\Omega} \chi \theta(x) u_{l k, k}^{*}\left(x^{\prime}, x\right) d \Omega(x)
$$

In DRM changes in temperature $\theta(x)$ are expressed in terms of known co-ordinate functions $f^{\prime}$, which are also temperature fields:

$$
\theta \approx \sum_{j=1}^{N+L+A} f^{j} \beta^{j}
$$

where $j$ are collocation points ( $N$ on the contour and $L$ in the domain, see Figure 1$), \beta^{j}$ is a set of initially unknown coefficients and $A$ augmentation functions are used to enrich the approximation.

The application of Navier equation to the generic function $f^{\prime}$, gives the corresponding particular solution $\hat{u}_{m k}^{j}$. Then, integrating (8) by parts, introducing (9) and suppressing $x^{\prime}$ and $x$ for convenience, gives 
$c_{l k}^{i} u_{k}^{i}+\int_{\Gamma} p_{l k}^{*} u_{k} d \Gamma-\int_{\Gamma} u_{l k}^{*}\left(p_{k}-\chi \theta n_{k}\right) d \Gamma=\sum_{j=1}^{N+L+A}\left[c_{l k}^{i} \hat{u}_{m k}^{i j}+\int_{\Gamma} p_{l k}^{*} \hat{u}_{m k}^{j} d \Gamma-\int u_{l k}^{*}\left(\hat{p}_{m k}^{j}-\chi f_{j} n_{m k}\right) d \Gamma\right] \beta_{m}^{j}$

where $\hat{p}_{m k}^{j}$ are the tractions corresponding to the particular solutions $\hat{u}_{m k}^{j}$.

The procedure for numerical solution of (10) follows that described for (7). Applying (8) to all boundary nodes gives rise to the following system

$$
\mathbf{H u}-\mathbf{G}^{\prime}=\left(\mathbf{H} \hat{\mathbf{u}}-\mathbf{G} \hat{p}^{\prime}\right) \beta
$$

The choice of approximation functions in (9) is somewhat arbitrary. Generally a radial basis function is used, such as $r, r^{2}, r^{3}$ or $r^{2} \log (r)$. These have shown to interpolate only in the neighbourhood of a particular point (local behaviour), so that global functions are also needed. For these last, terms in the Pascal triangle or global sine Pascal triangle are often employed, according to the specific type of applied external load.

\section{Implementation}

The devised optimisation algorithm follows the sequence mentioned in Section 2 for the BGM, now customized for BEM analysis.

First, an approximate design is proposed for the structure to be optimised. This is discretised working with quadratic isoparametric elements. Besides, internal collocation points are evenly distributed over the complete model domain and over the thin layer along the optimisation boundary (see Figure 1).

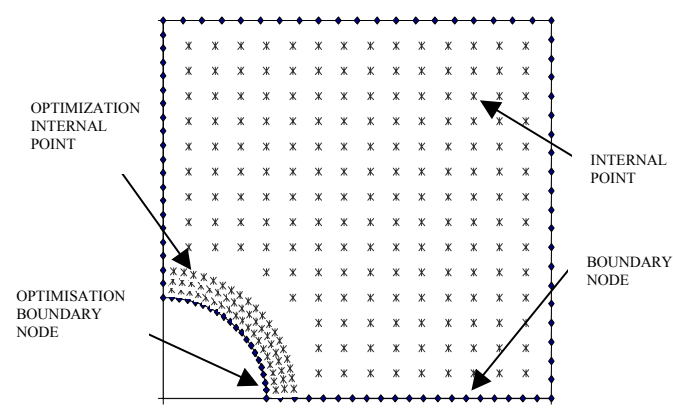

Figure 1. Generation of boundary and internal points on a given structure 
Then, a stress analysis is performed on this original geometry using the elastic BEM formulation In this way Von Mises stresses on the boundary nodes and on all of the internal collocation points are obtained.

These values of Von Mises stresses minus the reference stress (see (2)) are applied as a temperature field in DRM BEM formulation. In order to limit the swelling to the outermost layer of material, a temperature field different from null is specified only on the optimisation boundary nodes and the optimisation internal points. This computation supplies the displacements $u(x, y)$ and $v(x, y)$ along the optimisation boundary. In this work $r^{2} \log (r)$ was applied and terms up to the second degree in the Pascal triangle were chosen as augmentation functions (TAPT3 combination, as presented by Partridge and Sensale ${ }^{\mathrm{xvi}}$ ).

The optimisation boundary geometry is updated using exponential spline curves to avoid local wrinkles which could act as artificial stress raisers; and to generate a good quality BEM discretization (boundary nodes and internal collocation points) for the new geometry.

This whole process is repeated until acceptably low values of $\left(\sigma_{v m}-\sigma_{r e f}\right)$ are obtained.

\section{Examples}

Two problems were analysed to confirm the robustness of the proposed optimisation strategy. In the following sections the results for a square plate with a circular hole and a weld fillet are presented.

\subsection{Square Plate with Circular Hole}

The first problem introduced here is that of a square plate with a circular centred hole with remote loads applied in both directions $\sigma_{x}=45 \mathrm{MPa}$ and $\sigma_{y}=22.5 \mathrm{MPa}$ (see Figure 2). Due to

$$
\sigma_{y}=22.5 \mathrm{MPa}
$$

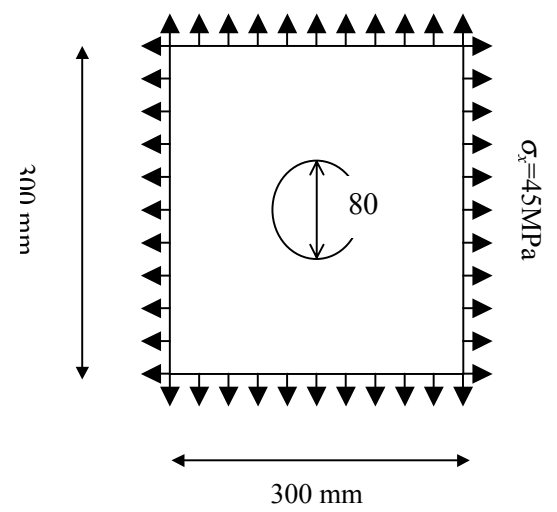

Figure 2. Square plate with circular hole. 
symmetry conditions only one quarter of the problem is considered, as has already been shown in Figure 1. The hole is the optimisation domain (note the thin layer of internal points parallel to this surface). The same problem was solved by Tekkaya and Güneri ${ }^{\mathrm{v}}$ using BGM and FEM. Following their analysis the reference stress was set to $\sigma_{r e f}=40 \mathrm{MPa}$ (nominal von Mises stress in the plate far from the hole), and $C=500$. The adopted value for Young modulus was $525 \mathrm{MPa}$.

Following Savin ${ }^{\text {xvi }}$ analytical optimisation predicts that in an infinite plate the circular hole will become elliptical and that the optimum geometry will be attained if and only if the quotient of maximum over minimum ellipse axis equals $\sigma_{x} / \sigma_{y}$, that is, 2 in this case. At the same time, Muskhelishvili ${ }^{\text {xvii }}$ analytically infers that the minimum von Mises stress attainable in an infinite plate with a circular hole and these boundary conditions is $\sigma_{v m}^{\text {minimum }}=\sigma_{x}+\sigma_{y}$, that is, $\sigma_{v m}^{\text {minimum }}=67.5 \mathrm{MPa}$ in this case.

The evolution of the normalized von Mises stresses $\left(\sigma_{v m} / \sigma_{r e f}\right)$ is plotted in Figure 3 as a function of the position (degrees) in the quarter of the hole. Also included as a reference are the results by Tekkaya and Güneri ${ }^{\mathrm{v}}$ for the original circular geometry. It can be observed that for the original geometry the peak value corresponds to the vertical edge $\left(\theta=0^{\circ}\right)$, while the minimum coincides with the horizontal edge $\left(\theta=90^{\circ}\right)$, as the applied load in the $y$-direction is half the applied load in the $x$-direction $\left(\sigma_{x} / \sigma_{y}=2\right)$. After five loops the normalized von Mises stresses are approximately uniform in the hole. This fact shows that the optimum uniform stress has been reached. At the same time, the stress value is close to $70 \mathrm{MPa}$, which agrees with Muskhelishvili's analysis, referenced above.

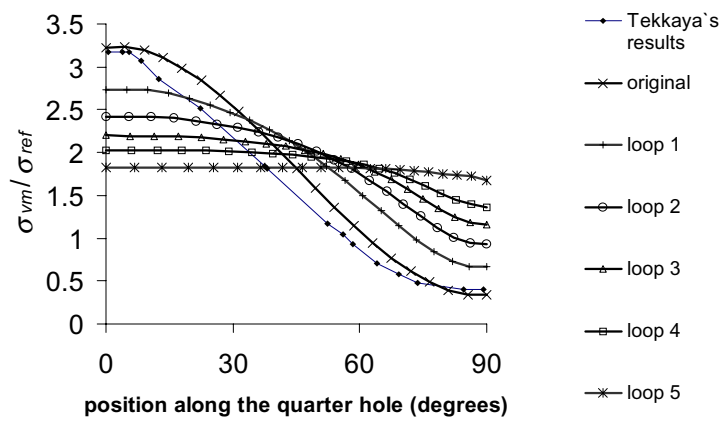

Figure 3. Evolution of the normalized von Mises stresses along the quarter of a hole during the optimisation. 
The evolution from circular to elliptical of the optimisation boundary can be seen in Figure 4. In the final geometry the quotient of maximum over minimum ellipse axis equals 2.00058 , in complete agreement with the theoretical predictions for an infinite plate, as stated above. Changes in shape follow variations in von Mises stresses. Both variations in stresses and geometry are illustrated in Figure 5. It is easy to see how the peak stress at $\theta=0^{\circ}$ is lowered

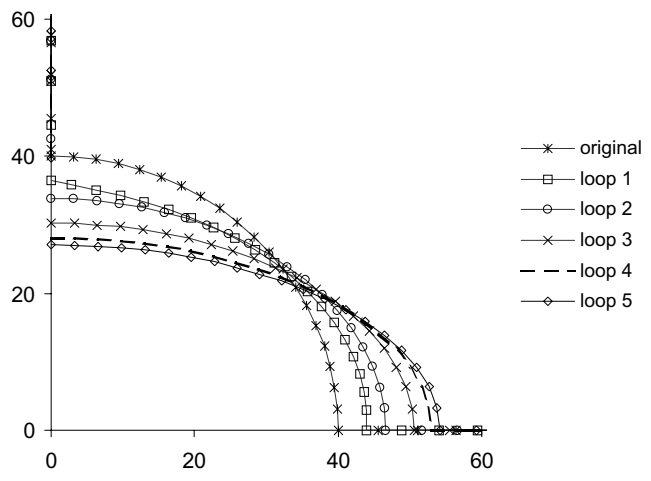

Figure 4. Evolution of hole profile as the optimisation progresses.

through the swelling of the area, while regions with stresses lower than the reference value $\left(\theta=90^{\circ}\right)$ shrink in order to increase the load level.

\subsection{Weld Fillet}

The last problem presented herein consists in a weld fillet, illustrated in Figure 6. A uniform stress $\sigma=10 \mathrm{MPa}$ is applied in the horizontal direction. The optimisation boundary is indicated with a thick line. Reference stress was chosen as $\sigma_{\text {ref }}=10 \mathrm{MPa}$ and $C=400.36$ elements and 198 internal points were used. The adopted value for Young modulus was $525 \mathrm{MPa}$. The same problem was solved by Li et $a l^{\mathrm{vi}}$ by means of Sensibility Analysis with FEM. 


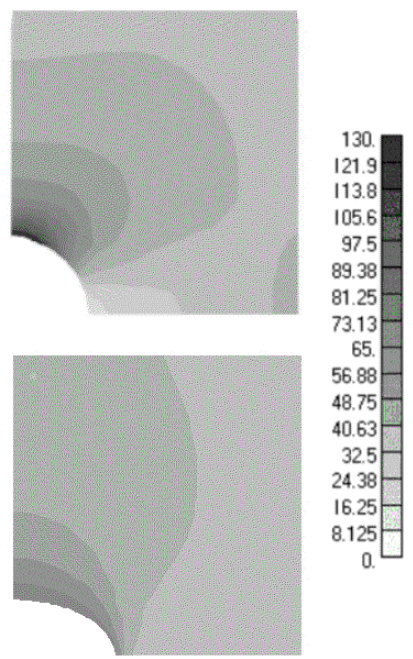

Figure 5. Distribution of von Mises stresses for the initial and final geometry

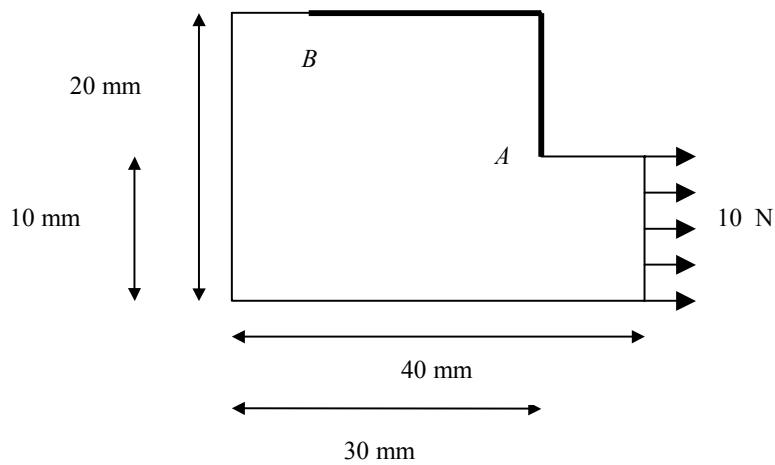

Figure 6. Weld fillet. 
Twenty-six optimisation loops were necessary in this case. Figure 7 illustrates the evolution of normalized von Mises stresses along the optimisation boundary, where the origin of the abscissas corresponds to position $A$, and 1 to position $B$ in Figure 6 . Note that with the exception of point $A$, the stress level on most of the optimisation boundary is bellow the reference value for the original configuration. As the optimisation progresses the thin layer shrinks, what results in a general augmentation in the stress level. Except in the region close to point $B$ where stresses can only be null, the final configuration shows a normalized stress distribution approximately equal to the reference stress, fact that confirms that the optimisation procedure has been fulfilled.

The evolution of the model shape is shown in Figure 8 for selected loops. Figure 9 illustrates the corresponding results by $\mathrm{Li}$ et $a l^{\mathrm{vi}}$. Note that both methods generate the same final geometry. Finally Figure 10 presents the initial and the final resultant geometry with the corresponding stress distributions.

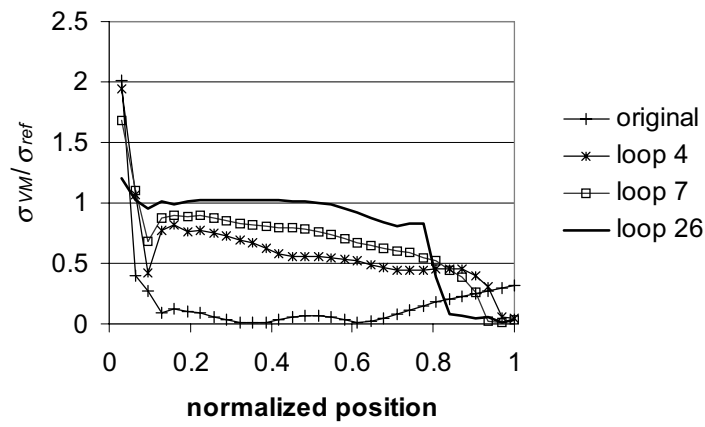

Figure 7. Evolution of normalized von Mises stresses as a function of the normalized position in the optimisation domain. 


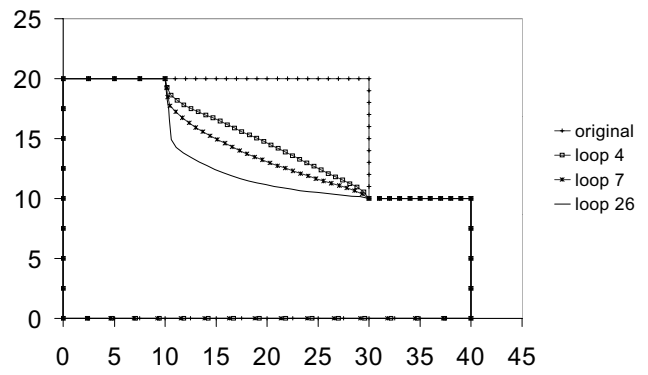

Figure 8. Evolution of fillet geometry as the optimisation progresses.
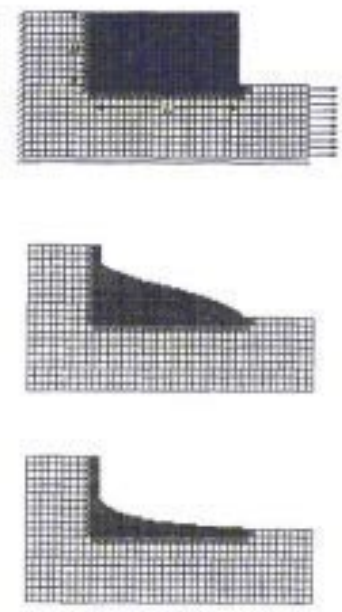

Figure 9. Evolution of fillet geometry as the optimisation progresses, reported by $\mathrm{Li}$ et al. 


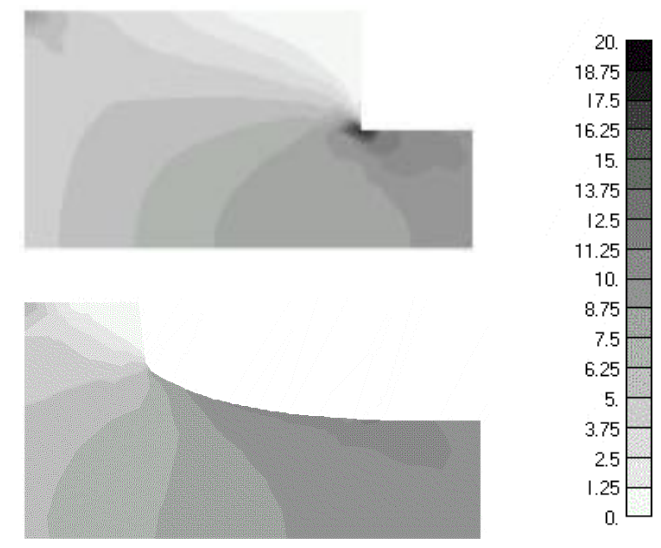

Figure 10. Distribution of von Mises stresses in the weld fillet for the initial and final configurations.

\section{Conclusions}

A numerical evolutionary procedure for the structural shape optimisation of two-dimensional problems is presented in this work. The proposed procedure is based in the Biological Growth Method (BGM) and was implemented using the Boundary Element Method (BEM). Two BEM formulations were employed in this work: the standard for two-dimensional elastostatics for the stress analysis, and the Dual Reciprocity Method (DRM) which was used to model the swelling or shrinking of the material.

BEM has proved to be an excellent analysis technique in this kind of problems. The optimisation of a shape problem by BEM, as described in this work, did not require the discretization of the model domain, neither for the stress or the swelling/shrinking analyses. This feature made the remeshing an easy task. Very accurate values of both domain stresses and displacements could be also obtained when using BEM.

BGM has proved to be a simple and effective method to obtain homogeneously distributed surface stresses. Besides, the optimisation method based on it was easy to implement. The versatility of the proposed methodology was illustrated by a series of examples, and results were compared to those reported in the bibliography. Excellent results were obtained for all cases showing the efficiency and effectiveness of the implementation. Coupling the BGM with BEM makes full use of the advantages of both methods. 


\section{Acknowledgements}

This work was founded by grant PICT 12-04586 of Agencia de Promoción Científica de la República Argentina, and the Comisión Sectorial de Investigación Científica de la Universidad de la República de Uruguay.

Carolina Wessel wants to express her thanks to the Universidad Austral for its support in her research activities.

\section{References}

${ }^{\mathrm{i}}$ A. D. Belegundu, and T. R. Chandrupatla, Optimization concepts and applications in engineering. New Jersey, USA: Prentice Hall (1999).

ii C. Mattheck, "Design and growth rules for biological structures and their application to engineering", Fatigue Fract. Engng. Mater. Struct., 13/5, 535-550 (1990).

${ }^{\text {iii }}$ C. Mattheck and S. Burkhardt, "A new method of structural shape optimisation based on biological growth", Int. J. Fatigue, 12/3, 185-190 (1990).

iv J. L. Chen and W. C. Tsai, "Shape optimization using simulated biological growth approaches", AIAA journal, 31/11, 2143-2147 (1993).

${ }^{v}$ A.E. Tekkaya and A. Güneri, "Shape optimization with the biological growth method: a parameter study". Engng. Comp, 13/8, 4-18 (1998).

${ }^{v i}$ Q. Li, P.S. Grant, O.M. Querin and Y.M. Xie, "Evolutionary shape optimization for stress minimization", Mech. Res. Comm, 26/6 657-664 (1999).

${ }^{v i i} \mathrm{P}$. Chaperon, "Shape optimization of damage tolerant structures for maximum residual static strength and fatigue Life", BENCHmark, 01, 14-16 (2001).

viii M. R. Baron and R. J. Yang, "Boundary integral equations for recovery of design sensitivities in shape optimization". J AIAA. 26/5 589-94 (1988).

ix J. H. Kane and S. Saigal, "Design-sensitivity of solids using BEM". Engng. Mech.114(10) 1703-22 (1988).

" S. C. Mellings and M. H. Aliabadi, "Flaw identification using boundary element method". Int. J. Num. Mech. Engn.38 399-419 (1995).

${ }^{x i}$ R. Cai, S. Cai, X. Yang and F. Lu, "A novel method of structural shape optimization coupling BEM with an optimization method based on biological growth", Struct. Opt, 15, 296-300 (1998).

${ }^{x i i}$ C. Mattheck and H. Moldenhauer, "An intelligent cad-method based on biological growth", Fatigue Fract. Engng. Mater. Struct, 13/1, 41-51 (1990).

xiii C.A. Brebbia and J. Domínguez, Boundary Elements: An Introductory Course. London, Southampton, UK: Computational Mechanics Publications Mc-Graw Hill Company (1992).

${ }^{\text {xiv }}$ P.W. Partridge, C.A. Brebbia and L.C. Wrobel, The dual reciprocity boundary element method. Southampton, UK: Computational Mechanics Publications (1992).

${ }^{x v}$ M.H. Aliabadi and D.P. Rooke, Numerical Fracture Mechanics. Southampton, UK: Computational Mechanics Publications and Kluwer Academic Publishers (1992). 
${ }^{x v i}$ P. W. Partridge and B. Sensale, "Hybrid approximation functions in the dual reciprocity boundary element method". Comm. Num. Mech. Engng. 13, 83-94 (1997).

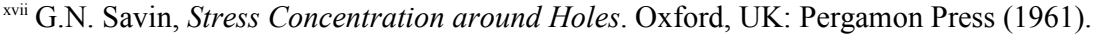

